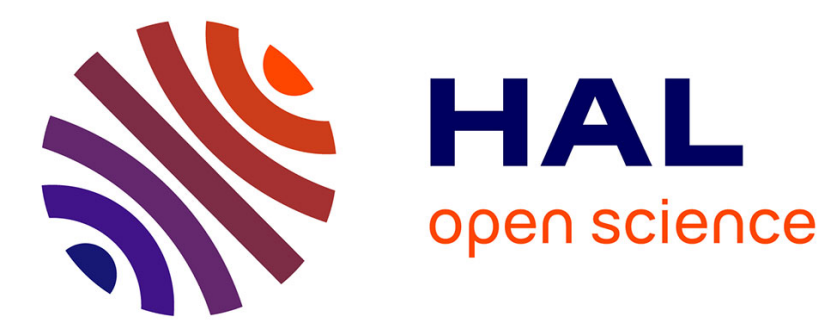

\title{
Hydrodynamics experiments with soap films and soap bubbles: A short review of recent experiments
}

H. Kellay

\section{To cite this version:}

H. Kellay. Hydrodynamics experiments with soap films and soap bubbles: A short review of recent experiments. Physics of Fluids, 2017, 29 (11), pp.111113 (1-10). 10.1063/1.4986003 . hal-01653622

\section{HAL Id: hal-01653622 \\ https://hal.science/hal-01653622}

Submitted on 1 Dec 2017

HAL is a multi-disciplinary open access archive for the deposit and dissemination of scientific research documents, whether they are published or not. The documents may come from teaching and research institutions in France or abroad, or from public or private research centers.
L'archive ouverte pluridisciplinaire HAL, est destinée au dépôt et à la diffusion de documents scientifiques de niveau recherche, publiés ou non, émanant des établissements d'enseignement et de recherche français ou étrangers, des laboratoires publics ou privés.

\section{다(1)(2)}

Distributed under a Creative Commons Attribution - ShareAlike| 4.0 International 


\title{
Hydrodynamics experiments with soap films and soap bubbles: A short review of recent experiments
}

\author{
H. Kellay \\ University of Bordeaux, CNRS, LOMA, UMR 5798, F-33405 Talence, France
}

\begin{abstract}
In this short review, I focus on recent experiments that benefit from the advantages offered by the twodimensionality of the flow in suspended thin liquid films to reconsider hydrodynamics problems which have resisted a full understanding. The first problem discussed here concerns friction drag in channel flows. The use of turbulent channel flows, using thin liquid films, allows measurements of friction drag as well as mean velocity profiles for flows with different spectral exponents. Is there a link between the spectral properties of the turbulence and the mean velocity profiles or the frictional drag? This is the first question considered. The second issue examined considers the long time dynamics of large scale vortices. These are obtained in half bubbles rendered "turbulent" through thermal convection. These vortices, which live in a quasi two-dimensional environment, have a long time dynamics where their vorticity goes through what seems to be a well-defined cycle with generic features.
\end{abstract}

\section{INTRODUCTION}

Since the seminal work of Kraichnan ${ }^{1}$ and Batchelor ${ }^{2}$ elucidating the role of spatial dimension on the properties of turbulence, the search was on for finding experimental systems to test and explore these properties. Experimental tests of the phenomenology of turbulence uncovered by this work had become a subject of interest and curiosity: Not one but two inertial ranges, new scaling laws, and a different direction for the energy cascade. Different systems have been studied since by different groups. ${ }^{3-6}$ Experiments have tested the predictions of this work through measurements of scaling laws for different quantities including energy and enstrophy spectra as well as the different moments of velocity or vorticity differences across different scales. ${ }^{4-6}$ Experiments did not stop at those tests and used quasi two-dimensional systems to study scalar dispersion and fluctuations, the dynamics and properties of large scale vortices, the interaction of a flow with different structures, etc. ${ }^{4-6}$ Work using electromagnetically driven flows in thin layers of fluid, flows driven by the excitation of surface waves, or flows in suspended liquid films had set the tone for such investigations and numerous studies have been carried out to examine issues as diverse as mixing, the role of shear on flows of thin liquid films, and the fluctuations of energy injection. Now original work is being carried out in different groups around the globe using such systems or variants of these. ${ }^{7-9}$ Here, in this short review, I will only focus on some very recent work carried out in collaboration with different groups whereby the twodimensional nature of the flows in suspended liquid films has been used to study the dynamics of large vortical structures or to bring new insight into friction drag. It is my purpose here to bring to the attention of the reader that new insight into some "old" issues can be obtained in quasi 2D systems.

\section{FRICTION DRAG IN TWO-DIMENSIONAL CHANNELS}

One such issue that has attracted the attention of experimentalists including my group, that of W. I. Goldburg in the US, the group of P. Chakraborty and G. Gioa in Japan, and that of N. Goldenfled in the US is the use of two-dimensional flows to study friction drag in 2D channels. The basic question can be stated simply: does friction drag in 2D show similar phenomenology as in 3D channels where much is known or are there fundamental differences between the two? As it turns out, the $2 \mathrm{D}$ case with its two different inertial ranges allows testing theories of friction drag in depth. Let me first introduce friction drag and how 2D flows may bring some insight into this problem which is related to how small scale properties and macroscopic properties of turbulent flows are linked.

Indeed, there are gaps in our fundamental understanding of turbulent flows in channels or pipes. Two aspects of such flows have been the subjects of extensive, but separate, research efforts: their macroscopic properties and their turbulent spectrum. The macroscopic properties of a turbulent flow are the properties of interest for practical and engineering purposes such as the frictional drag experienced by a flow past a wall ${ }^{10,11}$ and the mean velocity profile of the flow in a pipeline. ${ }^{10,11}$ The turbulent energy spectrum of the velocity fluctuations on the other hand is representative of the smallscale statistics of the flow as noted by Kolmogorov several decades ago. ${ }^{3,12-14}$ The link between the macroscopic properties and the turbulent spectrum has however remained unexplored. Only recently has experimental and theoretical work begun to focus on this issue and some of these results suggest that this link has important repercussions.

Interest and measurements of turbulent drag in pipes started almost a century ago. In the 1930s, Nikuradse carried out measurements of the friction factor of a turbulent pipe flow as a function of the Reynolds number $\mathrm{Re}=\mathrm{UR} / \mathrm{v}^{15}$ (here $\mathrm{U}$ 
is the mean velocity of the fluid, $\mathrm{R}$ is the radius of the pipe, and $v$ is the kinematic viscosity of the fluid) and as a function of $r / R$, the scale of the roughness of the pipe wall $r$ normalized by the pipe radius $\mathrm{R} .{ }^{16}$ This data set is usually shown in the form of plots of the so-called friction factor $f$ (which is a non-dimensional measure of the pressure drop in pipes: $f \sim \mathrm{R} \Delta \mathrm{P} / \rho \mathrm{U}^{2} \mathrm{~L}$ where $\Delta \mathrm{P}$ is the pressure drop over a length $\mathrm{L}$, $\rho$ is the density of the fluid, and $U$ is the mean velocity in the pipe) as a function of Re for different values of $r / R$. These data are shown in Fig. 1 where the asymptotic empirical scaling of Blasius, $f \sim \mathrm{Re}^{-1 / 4}$ for smooth pipes, and Strickler, $f \sim(\mathrm{r} / \mathrm{R})^{1 / 3}$ for rough pipes, is clearly visible.

A possible link between the small-scale properties of turbulence and the macroscopic response was originally based on these data and comes from two distinct studies. One development is the roughness-induced criticality theory of Goldenfeld, ${ }^{17}$ which derives the general form of the scaling law $f(\mathrm{Re}, \mathrm{r} / \mathrm{R})$ : to satisfy the asymptotic scalings $f \sim \mathrm{Re}^{-1 / 4}$ and $f \sim(\mathrm{r} / \mathrm{R})^{1 / 3}$, the friction factor must conform to the scaling law $f(\mathrm{Re}, \mathrm{r} / \mathrm{R})=\operatorname{Re}^{-1 / 4} \mathrm{~g}\left(\mathrm{Re}^{3 / 4}, \mathrm{r} / \mathrm{R}\right)$. This scaling law imposes that roughness must play an important role in any description of the turbulent wall bounded flows. The other development is the momentum-transfer theory of Gioia and Chakraborty, ${ }^{18}$ which derives an expression for $f(\mathrm{Re}, \mathrm{r} / \mathrm{R})$. This expression is consistent with the scaling law stated above and is a functional of the turbulent energy spectrum E(k). This latter development is the first attempt to give a physical argument explaining Nikuradse's data set. ${ }^{19}$

This momentum-transfer theory also yields the exponents of the asymptotic scalings of Blasius and Strickler as functions of the inertial-range exponent of the turbulent spectrum in 3D pipe flows (the spectral exponent of Kolmogorov, 5/3). The momentum transfer theory is especially useful because it can make precise predictions for macroscopic observables, such as the friction factor or the mean velocity profile, and relate their functional forms to the energy spectra.

These recent developments can, in particular, be generalized to arbitrary spectra. For a spectrum of arbitrary inertialrange exponent $\alpha$ [recall that $\alpha$ is defined so that $\mathrm{E}(\mathrm{k}) \sim \mathrm{k}^{-\alpha}$ within the inertial range], momentum-transfer theory yields

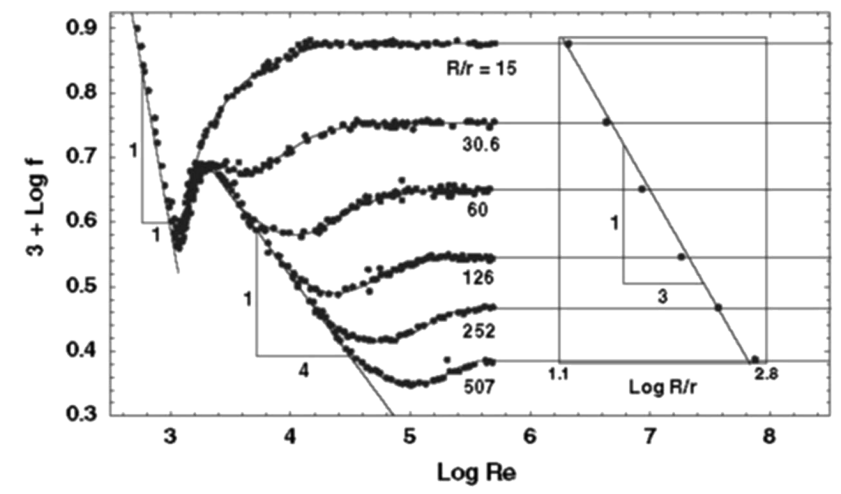

FIG. 1. Frictional drag $\mathrm{f}$ vs $\mathrm{Re}$ for pipe flows on walls of roughness $\mathrm{r} / \mathrm{R}$ : Nikuradse's data. Note the empirical Blasius and Strickler scalings. Adapted with permission from G. Gioia and P. Chakraborty, "Turbulent friction in rough pipes and the energy spectrum of the phenomenological theory," Phys. Rev. Lett. 96, 044502 (2006). Copyright 2006 American Physical Society. the generalized Blasius scaling ${ }^{17-20}$

$$
f \sim \operatorname{Re}^{(1-\alpha) /(1+\alpha)}
$$

and the generalized Strickler scaling ${ }^{17-20}$

$$
f \sim(\mathrm{r} / \mathrm{R})^{(\alpha-1) / 2} .
$$

These predictions explicitly use the value of the spectral exponent $\alpha$ and therefore can take into account differences in the statistical properties of the turbulence such as those which arise when going from 3D to 2D turbulence. A crucial aspect of this theory is that the wall stress is dominated by the characteristic velocity at the dissipation scale for smooth walls and the scale of the roughness for rough walls. The above relations 1 and 2 are then easily derived from the scaling of the characteristic velocity versus length scale and the scaling of the dissipation scale versus Re. Since the scaling of the characteristic velocities versus scale and that of the dissipation versus $\mathrm{Re}$ depend on $\alpha$, an explicit dependence on this parameter arises in the scaling of $f$.

A direct test of this powerful theoretical development would need to use different values of $\alpha$ but this is not possible in three-dimensional turbulent flows since the value of $\alpha=5 / 3$, the Kolmogorov value, is observed for most known turbulent flows. A way to change $\alpha$ is to use two-dimensional flows. ${ }^{1-6}$

We have recently performed experimental measurements of the friction factor in two-dimensional soap-film flows (where the two walls are smooth and the injection of vorticity is carried out using an array of several small cylinders) that exhibit the enstrophy cascade (a type of spectrum that does not exist in 3D flows, and for which $\alpha=3$ ) and verified that on smooth walls $f \sim \mathrm{Re}^{-1 / 2}$, in accord with (1) for $\alpha=3$. $^{21}$ Note that the generalized Blasius scaling of Eq. (1) and these experimental results cannot be explained using the classical theory of frictional drag formulated by Prandtl. ${ }^{11,21}$ Numerous variants and alternatives to the theory have been proposed. ${ }^{11,22,23}$ But the original theory of Prandtl and its variants are based on dimensional analysis and similarity assumptions, with no explicit reference to the turbulent spectrum.

In a second experiment, we have used a different way to generate turbulence in a soap film channel by using one rough and one smooth wall along with two different injection schemes (1 rod or an array of rods to generate turbulence) as illustrated in Fig. 2. This type of channel allows obtaining different spectral exponents. ${ }^{21}$ While the injection scheme using several small rods produces an enstrophy cascade type flow with an exponent $\alpha=3$. The spectral exponent $\alpha$ in the second configuration (using $1 \mathrm{rod}$ ) is close to $5 / 3$ as expected when an inverse cascade of energy occurs in two-dimensional flows. ${ }^{1,4}$ The exact reasons for obtaining an exponent of $5 / 3$ following the introduction of a rough wall and an injection of vorticity using a single large rod are not clear but it has been argued ${ }^{4}$ that wall roughness changes decaying turbulence into forced turbulence. The wall roughness forces the turbulence at small scales by injecting small scale eddies produced near the rough wall into the main flow favoring an inverse cascade. It is possible that using multiple rods with vortices, the size of the small rods renders observing the inverse cascade scaling more difficult. Nevertheless, the results for the energy spectra shown in Fig. 3 clearly point to two different scaling exponents. This 

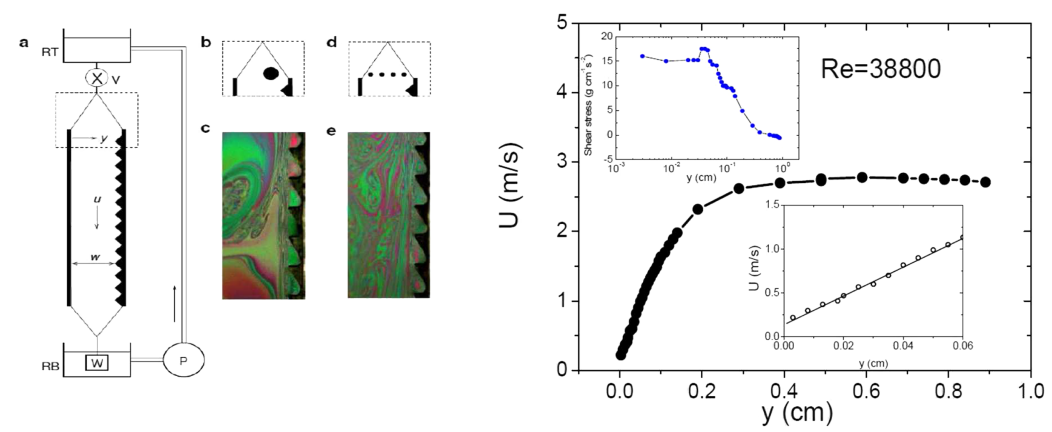

FIG. 2. (Left) The new soap film channel: (a) the film (in gray) hangs between two steel blades (shown in black) that are suspended by fishing wires kept taut by weight $\mathrm{W}$. One of the blades has a serrated edge (size of serrations $2 \mathrm{~mm}$ spaced by $4 \mathrm{~mm}$ ). The soap solution is injected from an upper reservoir and collected at a bottom reservoir. The gravity driven flow reaches mean velocities, tuned by varying the injection flux, between 0.5 and $3 \mathrm{~m} / \mathrm{s}$. The frictional drag is measured only on the smooth side of the channel. (b) Turbulence is generated by a rod of $6 \mathrm{~mm}$ diameter resulting in a flow with a $-5 / 3$ exponent. The turbulence can be made visible at a particular instant by (c) interference fringes in white light. (d) Turbulence is generated with the teeth of a comb (diameter $1 \mathrm{~mm}$ and spacing of $3 \mathrm{~mm}$ ) resulting in a flow with a -3 exponent. The turbulence can be made visible in (e) using interference fringes in white light. (Right) Velocity profile near the smooth wall: Lower inset shows a zoom on the viscous sub-layer where the velocity varies linearly with distance to the wall giving access to the shear rate at the wall. The upper inset shows the shear stress versus distance from the wall. Note that the shear stress increases as the wall is approached and shows a well-defined value near the wall. Adapted with permission from Kellay et al., "Testing a missing spectral link in turbulence," Phys. Rev. Lett. 109, 254502 (2012). Copyright 2012 American Physical Society.

new channel allows obtaining values of $\alpha$ of 3 or $5 / 3$ depending on the injection scheme used: an array of cylinders as in Fig. 2(d) produces $\alpha=3$ while a single rod [Fig. 2(b)] produces $\alpha=5 / 3$. This channel, therefore, allows testing the Blasius generalized scaling for two different spectral exponents $\alpha$ by simply switching from a rod to an array of cylinders. We recall in Fig. 2 (right panel) that the friction factor is obtained from measuring the shear rate near the smooth wall in the viscous sub-layer where the velocity varies linearly with distance from the smooth wall. In this sub-layer, the Reynolds stresses are estimated to be negligible so the wall stress is given directly by the shear stress near the wall: the wall shear rate multiplied by the viscosity as indicated in the inset of Fig. 2 (right panel). Figure 3 shows measurements of the friction factor $f$ versus
$R e$ for the two different channels. The friction factor scaling versus $\operatorname{Re}$ clearly depends on the value of $\alpha$ : as this value changes from 3 to $5 / 3$, the scaling of the friction factor changes from $\mathrm{Re}^{-1 / 2}$ to $\mathrm{Re}^{-1 / 4}$. This variation is in agreement with the expected variation from the generalized Blasius scaling.

The two experiments outlined above show the power of prediction of the simple spectral link theory discussed above, as it is capable of distinguishing between turbulent flows that differ in their spectral content. Considering the importance of linking the macroscopic properties to the fluctuations and of predicting friction drag for a variety of situations, it is essential to put this link to stringent tests beyond the two tests discussed above. Experiments are ongoing to examine the roughness dependence, for example, or the role of polymers on the friction a

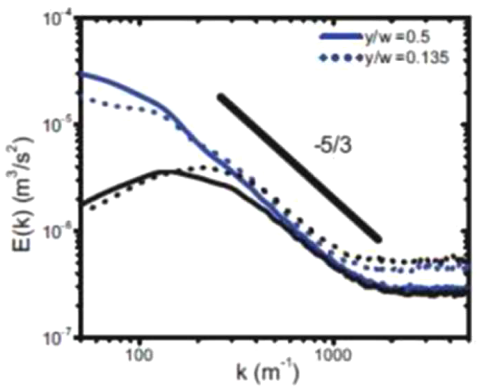

b

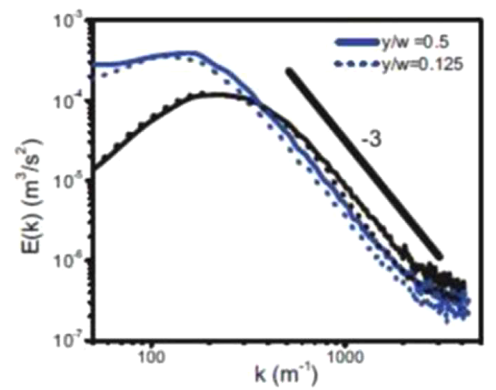

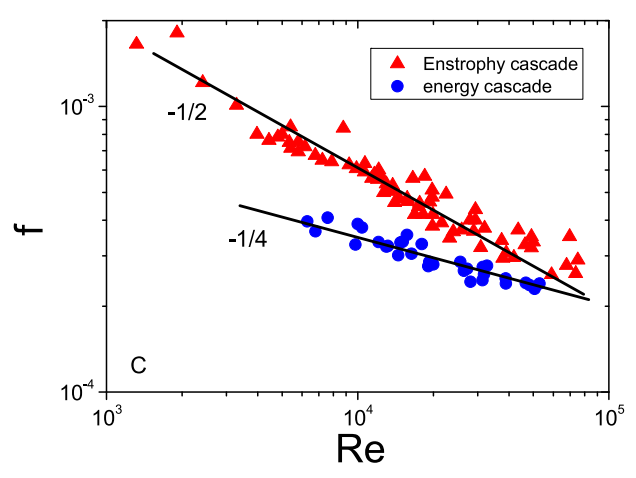

FIG. 3. Log-log plots of the energy spectrum [longitudinal (solid line) and transverse (dashed line) components are shown] in the two channels. (a) A rod at the inlet while (b) a comb at the inlet. Spectra are shown at two distances in the channel: near the center $y / w$ $=0.5$ and near the wall at $\mathrm{y} / \mathrm{w}=0.125$ ( $w$ is the width of the channel) showing the homogeneity of the turbulence in the transverse direction to the flow. (c) Friction factor variation versus the Reynolds number for the two flow configurations of Fig. 4. Note the difference in the exponent from $-1 / 2$ to $-1 / 4$ as predicted when the cascade switches from an enstrophy cascade to an energy cascade. (a) and (b) are reproduced with permission from Kellay et al., "Testing a missing spectral link in turbulence," Phys. Rev. Lett. 109, 254502 (2012). Copyright 2012 Americal Physical Society. (c) Part of the data in this figure is adapted from Ref. 24. 
drag. As mentioned above, direct predictions for the roughness dependence of the friction factor are at hand and need to be tested explicitly. Further, the use of polymers reduces drag and even if a large literature is devoted to this problem, no consensus exists as to its origin. Both issues can benefit from the ease of experimentation offered by these films and from the two-dimensionality of the flow which allows measuring all components of the velocity, the vorticity, and the local thickness of the film.

\section{MEAN VELOCITY PROFILES IN 2D TURBULENT CHANNEL FLOWS}

A related issue to friction drag concerns the functional form of the Mean Velocity Profiles (MVPs) in a turbulent channel or pipe flows. As is well known now in 3D turbulent pipe flows, the mean velocity profiles have universal features with a structure that is dependent on the distance from the wall. Three regions dominate the mean velocity profile: a viscous sublayer where the viscosity dominates the flow near the wall, a logarithmic layer farther out, and a buffer zone making the link between the viscous layer and the logarithmic zone. Again and considering the importance of the structure of the velocity profiles in setting the friction drag, we set out to determine whether such a universal structure has any relevance to $2 \mathrm{D}$ channel flows.

Turbulent flows over solid boundaries are ubiquitous, whether in natural or industrial settings. ${ }^{10,11}$ For threedimensional (3D) wall-bounded turbulent flows, Prandtl, in 1925 , postulated the dominant role of the wall shear stress (which slows down the flow near the wall due to the no slip boundary condition) to write down the law of the wall for the mean velocity profile (MVP). ${ }^{10}$ The near-wall region can be divided into three zones. Starting from zero velocity at the wall, the mean velocity linearly increases with distance from the wall in the viscous sublayer. In the buffer layer, this velocity continues to increase but at a smaller rate before changing into a logarithmic variation with increasing distance from the boundary. The logarithmic variation, known as the log law, was derived by von Karman in 1930 and is widely considered to be a fundamental result in the study of $3 \mathrm{D}$ turbulent wall bounded flows.

The law of the wall for the mean velocity profile, $\langle\mathrm{U}(\mathrm{y})\rangle$ [where $\mathrm{U}(\mathrm{y})$ is the longitudinal velocity at a distance $y$ from the wall, and \langle\rangle denotes averaging over time] is generally written in terms of the wall variables, $\mathrm{U}^{+}=\langle\mathrm{U}(\mathrm{y})\rangle / \sqrt{ } \tau_{\mathrm{w}} / \rho$ and $\mathrm{y}^{+}$ $=\mathrm{y} /\left(\nu \sqrt{ } \rho / \tau_{\mathrm{w}}\right)$, where $\tau_{\mathrm{w}}$ is the wall shear stress, $\rho$ is the density of the fluid, and $v$ its kinematic viscosity. This law is written as follows: $\mathrm{U}^{+}=\mathrm{f}\left(\mathrm{y}^{+}\right)$, where the functional form of $\mathrm{f}$ remains unspecified. In the viscous sublayer, $\mathrm{U}^{+}=\mathrm{y}^{+}$. In the log layer $\mathrm{U}^{+}=(1 / \kappa) \ln \left(\mathrm{y}^{+}\right)+\mathrm{B}$, where $\kappa$ is the von Karman constant and $\mathrm{B}$ is the offset of the log law. At high Reynolds numbers $(\mathrm{Re})$, the log region spans most of the spatial domain of the law of the wall, and, as Prandtl demonstrated, for a given Re the log law can be used to predict the wall shear stress. ${ }^{10}$ Precise measurements of the two parameters, $\kappa$ and $B$, are thus crucial to estimate the wall shear stress. In computing the frictional drag in pipe flows, or dissipation over different engines, these two parameters need to be known with high precision.

Numerous studies have shown that at high Re, these parameters are independent of Re, and that for different types of flows, the variation in $\kappa$ is within $10 \%$ and the variation in $\mathrm{B}$ (for flows over smooth walls) is within $25 \% .^{10,25,26}$ These observations indicate that the log law may well be a universal feature of 3D wall-bounded turbulent flows. ${ }^{27}$

The derivation of the law of the wall and the log law is based primarily on dimensional analysis and similarity arguments. ${ }^{10}$ These laws are independent of the dimension of the flow and the underlying turbulent spectrum. While 3D flows allow for only one turbulent spectrum (the energy cascade), two-dimensional (2D) turbulent flows allow two different turbulent spectra (the enstrophy cascade and the inverse energy cascade $)^{1-6}$ as we have seen above for channel flows. In contrast to 3D, there was no experimental study of the MVP in 2D or quasi-2D wall-bounded turbulent flows before. We therefore set out to study experimentally the near-wall MVP of quasi$2 \mathrm{D}$ turbulent channel flows. ${ }^{28}$ As in the previous friction drag measurements, the channels used allow for either energy cascade dominated flows or enstrophy cascade dominated flows with two different spectral exponents. Does the velocity profile depend on any way on the type of spectrum used? Are velocity profiles dependent on spatial dimension? These are the two questions we set out to answer.

To test the validity of the law of the wall and the log law, we measured MVPs for different Re and analyzed them in wall coordinates, $\mathrm{U}^{+}$vs. $\mathrm{y}^{+}$. In Fig. 4, we plot the MVPs for both turbulent spectra in semi-log coordinates. For each spectrum type, the MVPs corresponding to different Re collapse onto a universal curve in the near wall region confirming the validity of the law of the wall, $\mathrm{U}^{+}=\mathrm{f}\left(\mathrm{y}^{+}\right)$. (Farther away, the MVPs for different Re deviate from the universal curve, entering the domain where this law no longer holds.) Examining this universal curve we note the following: in the viscous sublayer, $\mathrm{U}^{+}=\mathrm{y}^{+}$; in the buffer layer, the curve tends toward
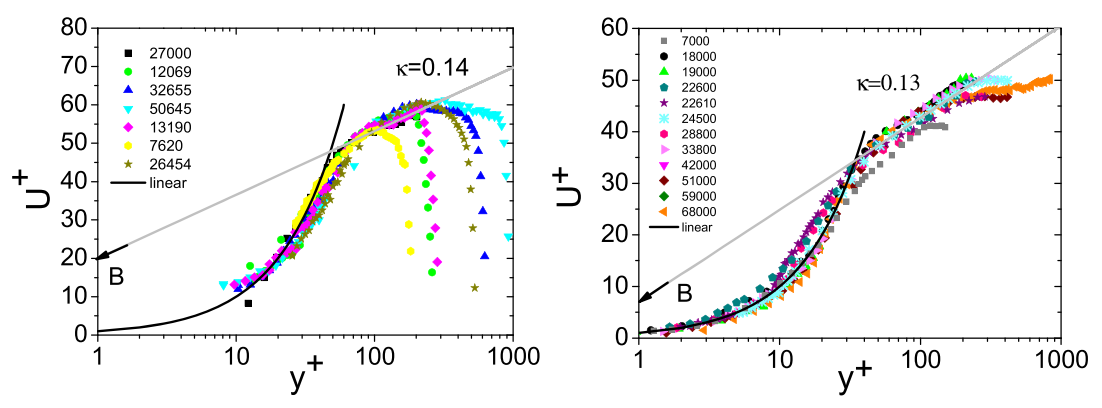

FIG. 4. Velocity profiles for the two different cascades (left: energy cascade and right: enstrophy cascade): wall units have been used here and data from different Reynolds numbers (given in the legends) are rescaled together. The data in this figure are adapted from Ref. 28. 

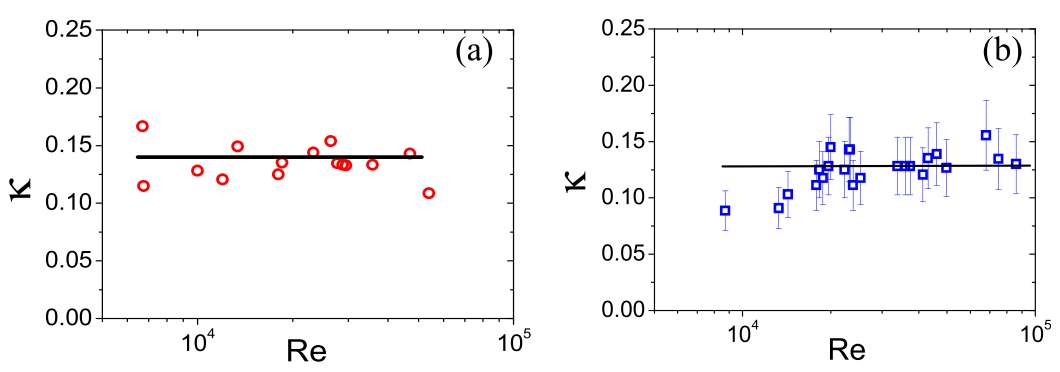

FIG. 5. The value of kappa versus Re for the two different cascades: (a) inverse cascade, (b) enstrophy cascade. Adapted with permission from Samanta et al., "Scaling of near-wall flows in quasi-two-dimensional turbulent channels," Phys. Rev. Lett. 113, 024504 (2014). Copyright 2014 American Physical Society. the logarithmic region; and in the log layer (which in semi$\log$ coordinates is a straight line $), \mathrm{U}^{+}=(1 / \kappa) \ln \left(\mathrm{y}^{+}\right)+\mathrm{B}$, thus validating the $\log$ law. These results appear to suggest an interesting prospect: that the law of the wall and the log law are not only universal features of 3D wall-bounded turbulent flows but also of quasi-2D wall-bounded turbulent flows. There are, however, two differences concerning the parameters of the log law.

First, consider the von Karman constant, $\kappa$. To estimate the value of $\kappa$, we take each MVP (such as the ones shown in Fig. 4) and replot as $\mathrm{y}^{+} \mathrm{dU}^{+} / \mathrm{dy}^{+}$vs. $\mathrm{y}^{+}$, wherein we look for a plateau, which, according to the log law, corresponds to $1 / \kappa$. For each type of spectrum, we plot the values of $\kappa$ vs. Re in Fig. 5. For flows with an inverse energy cascade, we find that $\kappa$ is approximately constant, with a mean value of 0.14 . For flows with an enstrophy cascade, we find that at large $\mathrm{Re}, \kappa$ is approximately constant, with a mean value of 0.13 , and that at lower $\mathrm{Re}, \kappa$ assumes smaller values. Within experimental uncertainty, we conclude that at high $\mathrm{Re}, \kappa$ in quasi-2D flows is independent of the turbulent spectra. This value of $\kappa$ is markedly different from that in 3D flows, where, to our knowledge, such small values of $\kappa$ have not been observed. ${ }^{10,25,26}$

Next, consider the offset of the log law, B. Limited by the experimental scatter, we estimate B from the MVPs shown in Fig. 4 using our above mentioned estimate of $\kappa$, we compute a best fit line for the log law, whose intercept at $\mathrm{y}^{+}=1$ corresponds to the value of B (see the arrows in Fig. 4). In contrast to $\kappa, \mathrm{B}$ depends on the turbulent spectrum: for flows with an inverse energy cascade, $\mathrm{B} \sim 20$; for flows with an enstrophy cascade, $\mathrm{B} \sim 7$. Interestingly, this latter value is close to $3 \mathrm{D}$ channel flows where $\mathrm{B}=5 .{ }^{10}$

Using quasi-2D turbulent flows in soap-film channels, we have tested that despite the differences with 3D wall-bounded flows in dimensionality and turbulent spectra, the near-wall MVP is composed of a viscous sublayer, a buffer layer, and a log layer, and obeys the law of the wall and the log law. The parameters of the log law are however very different: the von Karman constant $\kappa$ is independent of the turbulent spectra and is about one-third of its 3D counterpart; the offset of the log law B depends on the turbulent spectra and for the enstrophy cascade is close to its $3 \mathrm{D}$ counterpart. These results, which question the classical approach, await theoretical explanation.

\section{VORTICES IN SOAP BUBBLES}

In the above two examples, the two-dimensional nature of the flows and their ability to show different spectral properties have allowed testing new theories of friction drag as well as examine the validity of the log law of the wall under hitherto unexplored conditions of dimensionality and spectral properties. In the following, we use the $2 \mathrm{D}$ nature of flows in thin liquid layers to examine features related to large scale vortical structures and their dynamics.

\section{Interest and open questions}

Vortices are prominent features of fluid flows. They span length scales ranging from an insect's size ${ }^{29}$ to planetary dimensions. ${ }^{30}$ The understanding of the movement of these vortices, their structure, as well as their long time dynamics is important for different aspects whether at the level of small engines, natural turbulent flows, or planetary atmospheres. ${ }^{31}$ Vortices are found in turbulent flows at all scales, in the wake of a bluff body, or in atmospheric flows whether on Earth or on other planets. ${ }^{3,30}$ In the latter case, the observed single vortices are giant and very long lived. ${ }^{30,32}$ These vortices may also be short lived and capable of long distance travelling such as tropical cyclones (TC) on Earth. ${ }^{33,34}$ The exact structure of giant vortices and notably that of the great red spot on Jupiter or the dark spot on Neptune is still being examined, but some of their features have been measured: The two great spots are elliptical, for example, ${ }^{32,35,36}$ and tropical cyclones can be modeled with some success using a modified version of an ideal vortex known as the Rankine vortex. ${ }^{37}$ Nevertheless, when it comes to predicting their trajectories (as for TCs on Earth), the evolution of their intensity, or their lifetime, vortices resist description and modeling mainly due to the fact that their interactions with the environment are complex. ${ }^{34,38,39}$ Take, for example, the intensity of tropical cyclones (wind speed) and its temporal evolution. This is an issue that has been intensely studied over the past few decades with some success: thermodynamical models as well as simple coupled ocean atmosphere models seem to capture some of the essential features. ${ }^{38,40}$ However, its prediction remains a formidable task: several factors such as the interaction with the sea and the environment, the structure of the TC itself, as well as the role of rotation on the dynamics of such vortices ${ }^{39,41}$ have to be considered. It is therefore highly desirable to have simple experimental systems where the study of the generic properties of these vortices can be carried out. ${ }^{8,41-46}$

\section{Experimental realization of flows in curved geometry and observations}

In recent years, we have, in my group at Bordeaux, introduced a system where a half bubble heated from below is used to shed light on these problems. First of all, and to mimic atmospheric flows, the geometry needs to be spherical. Second, the 

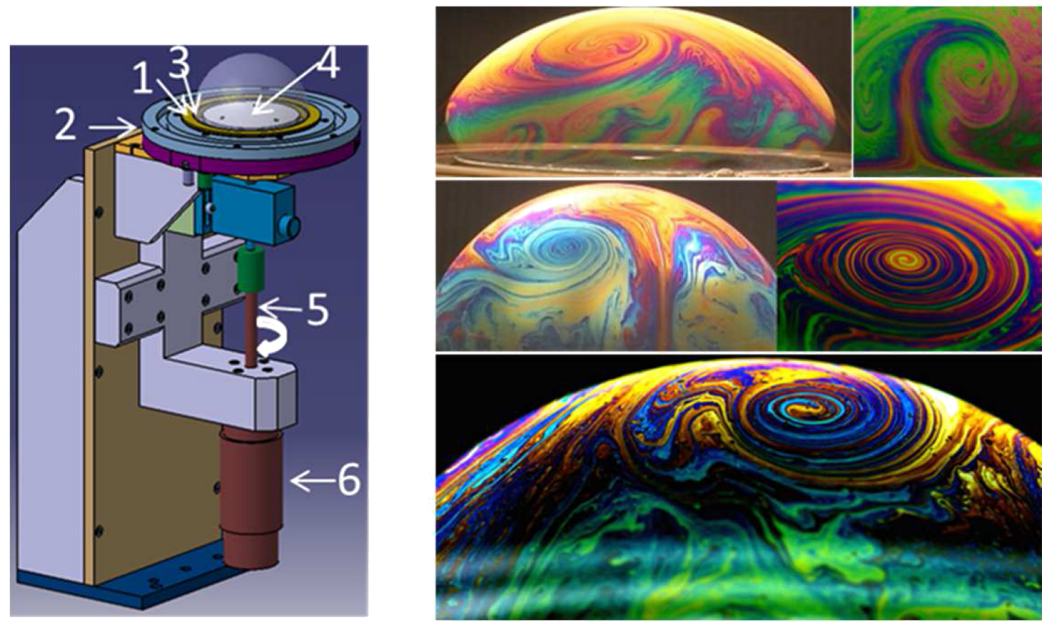

FIG. 6. The bubble: (left) setup: a brass disk (1) with a circular groove (3) can be rotated using a continuous motor (6) connected to it by a shaft (5). This disk is heated by the proximity of a hollow annulus (2) connected to a water circulation bath. The bubble is blown using the soap solution in the groove (3). The inner side of the brass disk is covered by a Teflon coating ( $2 \mathrm{~mm}$ thick) to minimize the heating of the air inside the bubble. The temperature at the equator of the bubble is set by the temperature of the water bath. (Right) Images showing the detachment of thermal plumes from near the equator and rising towards the pole, images of the full bubble with a vortex being formed by a large thermal plume and a zoom on a vortex, the colors are interference colors of white light being reflected by the thin water layer constituting the bubble.

system should be amenable to simple diagnostics and measurements. The half bubble system allows these features: the geometry is naturally curved, and the thin liquid film nature of the bubble makes it relatively easy to visualize the flow and its prominent features. Further, the system is simple enough to introduce rotation effects and therefore mimic, even more realistically, the sought after flows. In order to produce flow in such a system, a simple scheme consists of heating the bubble at the equator. Direct visualizations using color CCD cameras or infrared cameras allow extracting the thickness field as well as the temperature field. In addition, and either through following thickness inhomogeneities or through the use of Laser Doppler velocimetry (after seeding the solution with micrometer size particles), information about the velocity field or the velocity fluctuations can be obtained.

The system used is a half bubble heated from below $^{44,45,47,48}$ in a specially designed cell capable of rotating the bubble at different rates (see Fig. 6). Once formed, the bubble is subject to strong convection due to the heating at the base of the bubble. ${ }^{48}$ Images of the bubble with well-defined thermal plumes are shown in Fig. 6. By the way, this convection and its statistical properties can be studied in detail both for the velocity fluctuations using laser Doppler velocimetry and through infrared imaging for the temperature fluctuations. ${ }^{48}$ The most intriguing aspect is that after a short period, a large vortex may emerge. The emergence of vortices occurs both for bubbles not subjected to rotation and in bubbles subjected to rotation. The formation of these vortices occurs when a large plume, the result of the merging of a few smaller ones, rises to near the top of the bubble and forms a swirl as shown in Fig. 6 . This is only one possible way to form these vortices and our study did not allow us to uncover all the possible ways leading to their formation. Once they form, the large vortices are followed using video imaging. A large vortex of this kind is visible in Fig. 6. The vortex has a well-defined center or "eye" and develops as a spiral structure of dimensions between 1 and $2 \mathrm{~cm}$.

From velocity and vorticity profile measurements, it turns out that these vortices have profiles that are typical of a variety of known vortices. ${ }^{31}$ The ones examined here can be reasonably approximated by a Gaussian vortex. ${ }^{31}$ The azimuthal velocity profile of such a vortex obeys $\mathrm{V}(\mathrm{r})$
$=\Gamma /(2 \pi r)\left(1-\exp \left(-r^{2} / 4 \lambda^{2}\right)\right)$. Here $\Gamma$ is the circulation, $r$ is the distance from the center of the vortex, and $\lambda$ is a characteristic length. From the azimuthal velocity, we obtain the vorticity profile as $\omega(\mathrm{r})=\mathrm{V}(\mathrm{r}) / \mathrm{r}+\partial \mathrm{V}(\mathrm{r}) / \partial \mathrm{r}$. The Gaussian functional shape seems to capture the essential features of this vortex.

\section{Long time dynamics of vortices}

Besides the characterization of the structure of the observed vortices, we have also made measurements of the azimuthal velocity of the vortex over longer periods of time spanning several turnover times of the vortex. A typical turnover time of these vortices is a fraction of a second $(0.1 \mathrm{~s})$. An example of the long time dynamics of the azimuthal velocity is displayed in Fig. 7. Note that a long time variation can be observed in these measurements. This long time variation shows periods of constant low velocity and vorticity followed by an intensification period with higher velocities and vorticities. The measured velocity is for the same distance from

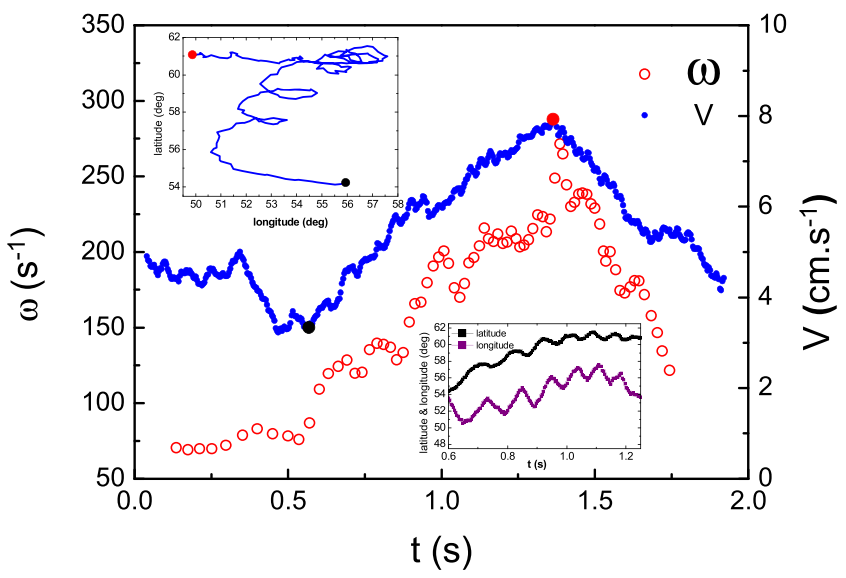

FIG. 7. Long time evolution of the azimuthal velocity and the vorticity of a single vortex, the upper inset shows the trajectory with visible trochoidal motion during the intensification phase marked by the red and black dots. The bottom inset shows the variation of the latitude and longitude of the vortex position versus time for the trajectory in the upper inset. Note the oscillations in the trajectory. Adapted from Meuel et al., "Intensity of vortices from soap bubbles to hurricanes," Sci. Rep. 3, 3455 (2013). Copyright 2013 Author(s), licensed under a Creative Commons Attribution 3.0 Unported License. 
the center so variation in the velocity is not related to the probe particle meandering towards the center of the vortex or moving away from it. Changes in the velocity or vorticity are solely due to the change of the vortex intensity in this case. The vortex followed here ends up reducing its velocity in the end and practically disappears in the background flow. We are not aware of other measurements of such long time dynamics of vortex properties in other systems or in theoretical work.

A curious aspect of our observations is that during the intensification period, the trajectory of the center of the vortex shows a trochoidal like motion as seen in Fig. 7: the vortex center wobbles a few times around its mean position. The period of this oscillation of the center of the vortex is roughly one turnover time that is comparable to the oscillation period observed for some tropical cyclones. This is seen for different vortices with the period of trochoidal motion being roughly the same and close to $0.1 \mathrm{~s}$. The link between this trochoidal motion and the intensity of the vortex in our case is not clear since this type of motion was observed both for the increasing intensity phase and during the decreasing intensity one. Different reasons have been proposed to explain this peculiar feature observed during the motion of some TC including instability of the core of the vortex or the existence of a double vortex structure giving rise to a periodic displacement of the vortex core with respect to its periphery. Our own observations do not allow a precise determination of the mechanisms at play here but our velocity measurements seem to exclude the double vortex hypothesis, and visualizations of the vortex do not seem to indicate large deformations of the core during this phase.

Let us come back to the intensification of vortices. In Fig. 8 we show several intensification events from the soap bubble as well as from numerical simulations of a half hemisphere heated at its equator and carried out by Bruneau and Fischer from the Institute of Mathematics in Bordeaux. ${ }^{47}$ Both

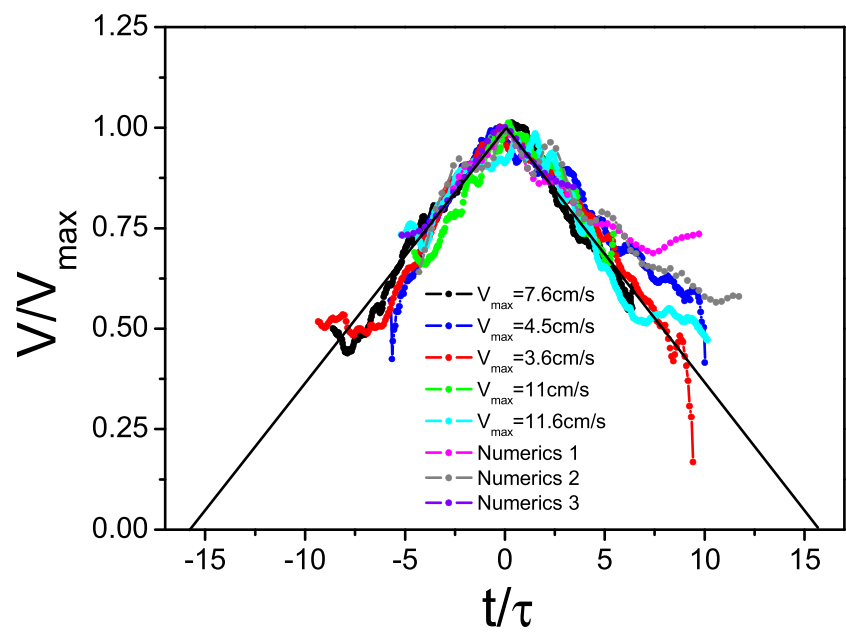

FIG. 8. Superposition of intensification events from the bubble vortices and from the numerics: vortex intensity from five different intensification events at different frequencies (of $0,0.2$, and $0.6 \mathrm{~Hz}$ ) versus time. Three intensification events from the numerics are also shown. The velocity axis has been normalized by the maximum velocity and the time has been normalized by a characteristic time $\tau$ and shifted so that the position of the maximum velocity is at zero. Adapted from Meuel et al., "Intensity of vortices from soap bubbles to hurricanes," Sci. Rep. 3, 3455 (2013). Copyright 2013 Author(s), licensed under a Creative Commons Attribution 3.0 Unported License. data sets, from five different intensification events in the soap bubble and three different events from the numerical simulations, show an intensification period where the velocity increases up to a maximum value we note $\mathrm{V}_{\max }$ followed by a decrease in intensity. The time scale in this figure has been shifted, so that $\mathrm{V}_{\max }$ occurs at time zero, and normalized by a characteristic time $\tau$. The velocity was simply normalized by $\mathrm{V}_{\max }$. An intriguing feature of this representation of the data is that a simple rescaling of the velocity and time axes leads to a collapse of all data sets onto a single universal curve suggesting that the intensification dynamics shows similar generic features for different vortices at different bubble rotation rates as well as for vortices from the numerical simulations. The time constant $\tau$ used for the rescaling of the data in this figure turns out to be roughly $0.07 \mathrm{~s}$ for the bubble vortices and for the vortices from the numerical simulations. This time scale is comparable to a turnover time. The mechanisms behind the intensification and decline of the vortices are however difficult to decipher.

The experimental system proposed here shows a variety of interesting features concerning the properties of vortices on the surface of the bubble: the existence of long lifetime vortices whose structure is well defined and whose long time dynamics shows intriguing properties such as intensification events and trochoidal motion. These features do not seem to have been observed before in other experimental systems so it is difficult to compare these observations to other laboratory experiments or to theoretical predictions.

\section{Comparisons with tropical cyclones}

While the vortices observed here are quasi twodimensional and therefore very different from natural giant vortices such as Tropical cyclones, some of the properties observed in our system (trochoidal motion and intensification) are also characteristic of Hurricanes and Typhoons. For the sake of comparison, we have examined whether such qualitative similarities can be made more quantitative. A particular feature we have examined in detail concerns the link between the intensification observed here and that of tropical cyclones. These are the only known vortices for which intensification as observed in our experiments is documented. In Fig. 9, we show the variation of the wind velocity $V(t)$ versus time $\mathrm{t}$ for a few hurricanes (data obtained from Ref. 49). Note that the normalization used above is useful to superimpose intensification data from different hurricanes. This figure shows that this rescaling works reasonably well for this data set. The only difference is the time constant (given in the figure) which varies but hovers around $6 \mathrm{~h}$.

We went a step further and superimposed data from other hurricanes alongside our data in Fig. 10. The curves from different tropical cyclones, from our vortices, and from our numerical simulations are superimposed in this representation suggesting that the variation of the velocity versus time is similar for such very different vortices spanning several orders of magnitude in length and time scales. In itself this result is perhaps not surprising since many different vortices may show an increase and a decrease in intensity. However, a notable feature is that the characteristic time $\tau$ needed to normalize the data turns out to be roughly constant and of order $6 \mathrm{~h}$ for TCs and 


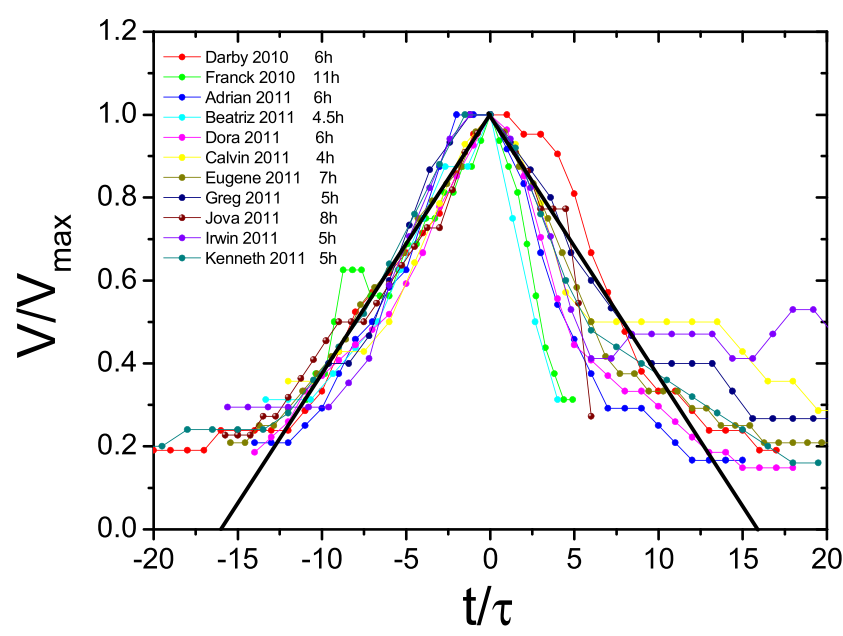

FIG. 9. Intensification for several hurricanes in the North Eastern Pacific. Here again the time and velocity axes have been normalized as in Fig. 8. The time constants are given along with the name of the TC in the figure. Adapted from Meuel et al., "Intensity of vortices from soap bubbles to hurricanes," Sci. Rep. 3, 3455 (2013). Copyright 2013 Author(s), licensed under a Creative Commons Attribution 3.0 Unported License.

$0.07 \mathrm{~s}$ for our vortices as shown in the inset. For the numerics, the value of $\tau$ is about $0.07 \mathrm{~s}$ for the three vortices shown in very good agreement with the experiments. Each system therefore seems to be characterized by a single mean time constant. While the exact meaning of this time constant is not clear at present, its order of magnitude points to roughly one turnover time for the experiments and the numerics, and roughly one turnover time for the tropical cyclones if the radius of hurricane force winds is considered. In order to test whether for tropical cyclones the time constant is robust and not just a coincidence, we have performed an analysis of a large ensemble of events. A time constant was obtained from superimposing all the data onto the same universal curve. The histogram of $\tau$ values obtained from an analysis of 171 TCs in the Atlantic and the Pacific basins is shown in Fig. 10. Note that the histogram is well defined suggesting that the value of $\tau$ has a mean of $6 \mathrm{~h}$ with a standard deviation of about $2 \mathrm{~h}$. Furthermore, and in Fig. 10, we have added data from a compilation of tropical cyclone intensity variation with time (over 56 storms in the Atlantic and 73 storms in the Pacific) obtained from Ref. 50. The normalization as above of this data set using a time constant of $6 \mathrm{~h}$ works reasonably well.

The superposition of the data shown in Fig. 10 suggests that a simple relation for the variation of the wind speed versus time can be written: $\mathrm{V}(\mathrm{t})=\mathrm{V}_{\max }\left(1 \pm\left(\mathrm{t}-\mathrm{t}_{\max }\right) / 16 \tau\right)$ or equivalently $\partial \mathrm{V}(\mathrm{t}) / \partial \mathrm{t}= \pm \mathrm{V}_{\max } / 16 \tau$ for the increase and the decrease in intensity before and after $t_{\max }$. Note that there are deviations for $t>t_{\max }$ (see Katrina case in Fig. 10) when the hurricane approaches landfall and the intensity decreases fast. From this relation, the maximum attainable wind speed $\mathrm{V}_{\max }$ is directly related to the rate of change of the velocity during the intensification period. Separate estimates of the velocity rate of change $\partial \mathrm{V}(\mathrm{t}) / \partial \mathrm{t}$ and the maximum speed $\mathrm{V}_{\max }$ agree with this relation as seen in the inset of Fig. 10. Further, since the temporal variation of the velocity turns out to be roughly linear, the duration of the intensification period or $\mathrm{t}_{\max }$ can be determined as it lasts
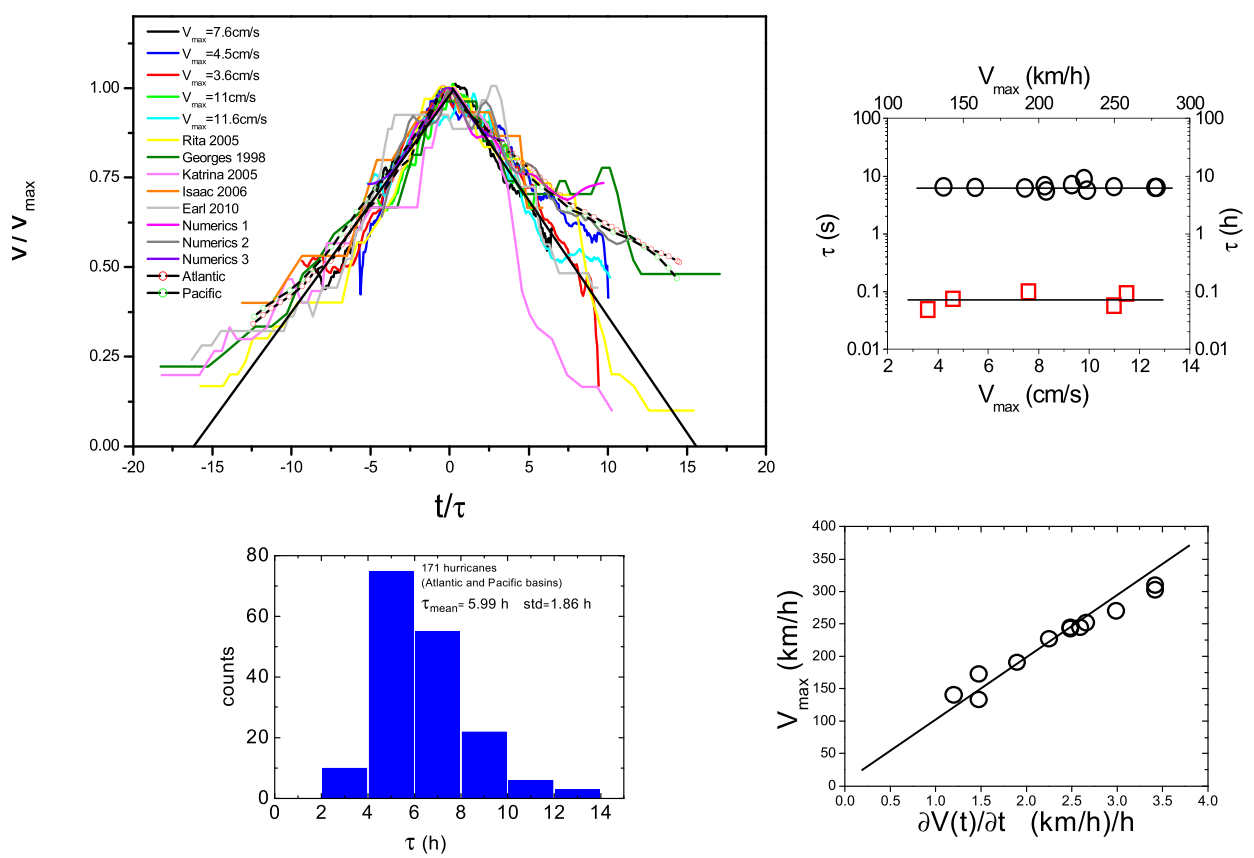

FIG. 10. Hurricane intensity from five different hurricanes in the Atlantic as well as two compilations extracted from Ref. 50 for the Atlantic and Pacific oceans and vortex intensity from five different intensification events versus time. Three intensification events are from the numerics. The upper right figure shows the characteristic time versus maximum velocity for the intensification events shown in the main figure and for a few additional hurricanes (Georges 1998 , Bill 2009, Rita 2005, Katrina 2005, Beta 2005, Helene 2006, Paloma 2008, Dolly 2008, Omar 2008, Isaac 2006, Earl 2010). The bottom right figure shows the linear relation between intensification rate and maximum velocity for a dozen hurricanes (different from the ones used in the upper right figure and the main figure: Opal 1995, Andrew 1992, Hugo 1989, Dean 1989, Gilbert 1988, Gloria 1985, Camille 1969, Igor 2010, Gordon 2006, Florence 2006, Dean 2007, Chris 1994). The bottom figure shows a histogram of the time $\tau$ obtained from an analysis of 171 tropical cyclones in the Atlantic and the Pacific oceans. The mean value of $\tau$ is $6 \mathrm{~h}$ with a standard deviation of $1.8 \mathrm{~h}$. Adapted from Meuel et al., "Intensity of vortices from soap bubbles to hurricanes," Sci. Rep. 3, 3455 (2013). Copyright 2013 Author(s), licensed under a Creative Commons Attribution 3.0 Unported License. 


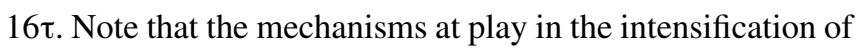
vortices here and of tropical cyclones are very different: our vortices are prone mostly to interactions with the background flow while tropical cyclones even though very extended in their lateral dimension have a full and complex three-dimensional structure which is essential to their dynamics. Nevertheless, the superposition of the data sets suggests that the dynamics of the intensification of vortices may have generic features that call for a full understanding.

\section{CONCLUSION AND PERSPECTIVES}

This short review brought together recent work on flows in thin liquid films, either in flat or curved geometries. In both cases, the quasi two-dimensional nature of the system allows to reconsider important fluid dynamics questions. Friction drag with different turbulent flows, and vortex properties in curved geometry have been revisited. These thin liquid films can be used to consider other problems such as the interaction of flows with different structures. Experiments on thin filaments, deformable structures, or flow induced oscillations have been examined. ${ }^{51-53}$ One interesting development by my group in collaboration with an Italian and a Swedish team focused on flow induced symmetry breaking in the problem of flow structure interaction. ${ }^{53}$ This development opens the way towards passive flow induced locomotion with implications for sedimentation and particle transport in turbulent flows. Other perspectives are open in the use of these films to study flows in porous like materials in the presence of numerous obstacles or flows with deformable walls. The half bubbles introduced here can be rotated in a simple setup opening the way to a full study of rotation effects on thermal convection or Marangoni convection in curved geometries. Our initial studies already indicate the suppression of turbulent fluctuations with the Rossby number and reduced heat flux. There are certainly other developments to come.

\section{ACKNOWLEDGMENTS}

This work has been carried out with several colleagues and students to whom I am indebted and thankful: W. I. Goldburg, X. L. Wu, N. Goldenfeld, P. Chakraborty, G. Gioia, C. H. Bruneau, P. Fischer, M. Bessafi, F. Ingremeau, T. Meuel, F. Seychelles, T. Tran, R. Cerbus, D. Samanta, S. Bagheri, and A. Mazzino.

${ }^{1}$ R. Kraichnan, "Inertial ranges in two-dimensional turbulence," Phys. Fluids 10, 1417 (1967).

${ }^{2}$ G. K. Batchelor, "Computation of the energy spectrum in homogeneous two-dimensional turbulence," Phys. Fluids 12, 233 (1969).

${ }^{3}$ U. Frisch and A. Kolmogorov, Turbulence: The Legacy of A. N. Kolmogorov (Cambridge University Press, 1995).

${ }^{4}$ H. Kellay and W. I. Goldburg, "Two-dimensional turbulence: A review of some recent experiments," Rep. Prog. Phys. 65, 845 (2002).

${ }^{5} \mathrm{P}$. Tabeling, "Two dimensional turbulence: A physicist approach," Phys. Rep. 362, 1 (2002).

${ }^{6}$ G. Boffetta and R. E. Ecke, “Two dimensional turbulence," Annu. Rev. Fluid Mech. 44, 427 (2012).

${ }^{7}$ H. Xia, D. Byrne, G. Falkovich, and M. Shats, "Upscale energy transfer in thick turbulent fluid layers," Nat. Phys. 7, 321 (2011).
${ }^{8}$ N. Francois, H. Xia, H. Punzmann, and M. Shats, "Inverse energy cascade and emergence of large coherent vortices in turbulence driven by Faraday waves," Phys. Rev. Lett. 110, 194501 (2013).

${ }^{9}$ C.-C. Liu, R. Cerbus, and P. Chakraborty, "Janus spectra in two-dimensional flows," Phys. Rev. Lett. 117, 114502 (2016).

${ }^{10}$ S. Pope, Turbulent Flows (Cambridge University Press, Cambridge, UK, 2000).

${ }^{11} \mathrm{H}$. Schlichting and K. Gersten, Boundary-Layer Theory (Springer, New York, USA, 2000).

${ }^{12} \mathrm{~A}$. N. Kolmogorov, "Local structure of turbulence in incompressible fluid at a very high Reynolds number," Dokl. Akad. Nauk SSSR 30, 299-302 (1941).

${ }^{13}$ A. M. Obukhov, "Energy distribution in the spectrum of turbulent flow," Dokl. Akad. Nauk SSSR 32, 22-24 (1941).

${ }^{14}$ K. R. Sreenivasan, "Fluid turbulence," Rev. Mod. Phys. 71, S383-S395 (1999).

${ }^{15} \mathrm{~J}$. Nikuradse, "Gesetzmassigkeiten der turbulenten strömung in glatten rohren," VDI-Forschungsh. 356, 1-36 (1932).

${ }^{16}$ J. Nikuradse, "Stromungsgesetze in rauhen rohren," VDI-Forschungsh. 361, 1-22 (1933).

${ }^{17}$ N. Goldenfeld, "Roughness-induced critical phenomena in a turbulent flow," Phys. Rev. Lett. 96, 044503 (2006).

${ }^{18} \mathrm{G}$. Gioia and P. Chakraborty, "Turbulent friction in rough pipes and the energy spectrum of the phenomenological theory," Phys. Rev. Lett. 96, 044502 (2006).

${ }^{19}$ J. J. Allen, M. A. Shockling, G. J. Kunkel, and A. J. Smits, "Turbulent flow in smooth and rough pipes," Philos. Trans. R. Soc., A 365, 699 (2007).

${ }^{20} \mathrm{G}$. Gioia, P. Chakraborty, and F. Bombardelli, "Rough-pipe flows and the existence of fully developed turbulence," Phys. Fluids 18, 038107 (2006).

${ }^{21}$ T. Tran, P. Chakraborty, N. Guttenberg, A. Prescott, H. Kellay, W. Goldburg, N. Goldenfeld, and G. Gioia, "Macroscopic effects of the spectral structure in turbulent flows," Nat. Phys. 6, 438 (2010).

${ }^{22} \mathrm{~W}$. George, "Is there a universal log law for turbulent wall-bounded flows?," Philos. Trans. R. Soc., A 365, 789 (2007).

${ }^{23}$ G. Barenblatt and A. Chorin, "A mathematical model for the scaling of turbulence," Proc. Natl. Acad. Sci. 101, 15023 (2004).

${ }^{24}$ H. Kellay, T. Tran, W. I. Goldburg, N. Goldenfeld, G. Gioia, and P. Chakraborty, "Testing a missing spectral link in turbulence," Phys. Rev. Lett. 109, 254502 (2012).

${ }^{25}$ I. Marusic et al., "Wall-bounded turbulent flows at high Reynolds numbers: Recent advances and key issues," Phys. Fluids 22, 065103 (2010).

${ }^{26}$ A. J. Smits, B. J. Mckeon, and I. Marusic, "High-Reynolds number wall turbulence," Annu. Rev. Fluid Mech. 43, 353 (2011).

${ }^{27}$ G. Gioia, N. Guttenberg, N. Goldenfeld, and P. Chakraborty, "Spectral theory of the turbulent mean-velocity profile," Phys. Rev. Lett. 105, 184501 (2010).

${ }^{28}$ D. Samanta, F. Ingremeau, R. Cerbus, T. Tran, W. I. Goldburg, P. Chakraborty, and H. Kellay, "Scaling of near-wall flows in quasi-two dimensional turbulent channels," Phys. Rev. Lett. 113, 024504 (2014).

${ }^{29}$ D. L. Hu, B. Chan, and J. W. M. Bush, "The hydrodynamics of water strider locomotion," Nature 424, 663 (2003).

${ }^{30}$ P. S. Marcus, "Numerical simulation of Jupiter's great red spot," Nature 331, 693 (1988).

${ }^{31}$ S. I. Green, Fluid Vortices (Kluwer Academic Publishers, The Netherlands, 1995).

${ }^{32}$ L. M. Polvani, J. Wisdom, E. Dejong, and A. P. Ingersoll, "Simple dynamical models of Neptune's great dark spot," Science 249, 1393 (1990).

${ }^{33}$ J. C. L. Chan and J. D. Kepert, Global Perspectives on Tropical Cyclones (World Scientific Publishing Co., 2010).

${ }^{34}$ K. E. Emanuel, "Tropical cyclones," Annu. Rev. Earth Planet. Sci. 31, 75 (2003).

${ }^{35}$ T. Liu, B. Wang, and D. S. Choi, "Flow structures of Jupiter's great red spot extracted by using optical flow method," Phys. Fluids 24, 096601 (2012).

${ }^{36}$ D. S. Choi, D. Banfield, P. Gierasch, and A. P. Showman, "Velocity and vorticity measurements of Jupiter's great red spot using automated cloud feature tracking," Icarus 188, 35 (2007).

${ }^{37}$ K. J. Mallen, M. T. Montgomery, and B. Wang, "Reexamining the near-core radial structure of the tropical cyclone primary circulation: Implications for vortex resiliency," J. Atmos. Sci. 62, 408 (2005).

${ }^{38}$ K. A. Emanuel, "Thermodynamic control of hurricane intensity," Nature 401, 665 (1999). 
${ }^{39}$ M. T. Montgomery and R. Smith, "Paradigms for tropical cyclone intensification,” Aust. Meteorol. Oceanogr. J. 64, 37 (2014).

${ }^{40} \mathrm{~K}$. Ooyama, "Numerical simulation of the life cycle of tropical cyclones," J. Atmos. Sci. 26, 3 (1969).

${ }^{41}$ R. K. Smith, C. W. Schmidt, and M. T. Montgomery, "An investigation of rotational influences on tropical-cyclone size and intensity," Q. J. R. Meteorol. Soc. 137, 1841 (2011).

${ }^{42}$ J. Sommeria, S. D. Meyers, and H. L. Swinney, "Laboratory model of a planetary eastward jet," Nature 337, 58 (1989).

${ }^{43}$ T. H. Solomon, W. J. Holloway, and H. L. Swinney, "Shear flow instabilities and Rossby waves in barotropic flow in a rotating annulus," Phys. Fluids A 5, 1971 (1993)

${ }^{44}$ F. Seychelles, Y. Amarouchene, M. Bessafi, and H. Kellay, "Thermal convection and emergence of isolated vortices in soap bubbles," Phys. Rev. Lett. 100, 144501 (2008).

${ }^{45}$ T. Meuel, G. Prado, F. Seychelles, M. Bessafi, and H. Kellay, "Hurricane track forecast cones from fluctuations," Sci. Rep. 2, 446 (2012).

${ }^{46}$ J. S. Turner and D. K. Lilly, "The carbonated-water tornado vortex," J. Atmos. Sci. 20, 468 (1963).
${ }^{47}$ T. Meuel, Y. L. Xiong, P. Fischer, C. H. Bruneau, M. Bessafi, and H. Kellay, "Intensity of vortices from soap bubbles to hurricanes," Sci. Rep. 3, 3455 (2013).

${ }^{48}$ F. Seychelles, F. Ingremeau, J. C. Pradere, and H. Kellay, "From intermittent to nonintermittent behavior in two dimensional thermal convection in a soap bubble," Phys. Rev. Lett. 105, 264502 (2010).

${ }^{49}$ See http://www.nhc.noaa.gov for "The National Hurricane Center" (last accessed March 5, 2013).

${ }^{50}$ K. E. Emanuel, “A statistical analysis of tropical cyclone intensity,” Mon. Weather Rev. 128, 1139 (2000).

${ }^{51}$ S. Childress, M. Shelley, and J. Zhang, "Fluid-structure interactions: Research in the courant institute's applied mathematics laboratory," Commun. Pure and Appl. Math. 65, 1697 (2012).

${ }^{52}$ A. Orchini, H. Kellay, and A. Mazzino, "Galloping instability and control of a rigid pendulum in a flowing soap film," J. Fluids and Struct. 56, 124 (2015).

${ }^{53}$ U. Lacis, N. Brosse, F. Ingremeau, A. Mazzino, F. Lundell, H. Kellay, and S. Bagheri, "Passive appendages generate drift through symmetry breaking," Nat. Commun. 5, 5310 (2014). 